\title{
El uso minifundista de la tierra como eje central del preacuerdo agrario entre el gobierno de Colombia y las FARC. Factibilidad preliminar teórica y práctica
}

The smallholding land use as central axis of the rural pre agreement between the Colombian government and the FARC. Preliminary theoretical and practical 


\title{
El uso minifundista de la tierra como eje central del preacuerdo agrario entre el gobierno de Colombia y las FARC. Factibilidad preliminar teórica y práctica ${ }^{1}$
}

\section{The smallholding land use as central axis of the rural pre agreement between the Colombian government and the FARC. Preliminary theoretical and practical feasibility}

\author{
Bernardo Congote Ochoa ${ }^{2}$, José A. Lizcano Caro ${ }^{3}$ \\ Universidad Antonio Nariño, Central Bogotá, Colombia \\ Universidad Distrital Francisco José de Caldas, Bogotá, Colombia
}

Artículo recibido en marzo de 2016; artículo aceptado en mayo de 2016

Citación del artículo: Congote, B. \& Lizcano, J. (2016). El uso minifundista de la tierra como eje central del preacuerdo agrario entre el gobierno de Colombia y las FARC. Factibilidad preliminar teórica y práctica.

I+D Revista de Investigaciones, 8(2), pp. 25 - 34

\begin{abstract}
Resumen
En Colombia la guerra civil no estaría justificada en desacuerdos estructurales entre las partes, al menos en lo relacionado con las soluciones al problema agrario. El artículo lo demuestra analizando el Punto Uno del preacuerdo de paz entre el gobierno de Colombia y las FARC en La Habana, denominado "Política de desarrollo agrario integral". La hipótesis a probar consiste en que el eje central del preacuerdo tiende a fomentar y proteger al minifundio como modo eficiente de explotación de la tierra y, por esta ruta, a perpetuar la pobreza rural manteniendo intacta esta causa objetiva de la violencia política. Primero, el artículo analiza y sintetiza el texto del preacuerdo comparándolo con las disposiciones contenidas en las Leyes agrarias 200/36 y 135/61, buscando precisar sus puntos de contacto más relevantes. Segundo, conceptúa comparadamente que no se observan diferencias cualitativas ni cuantitativas entre lo acordado entre Gobierno y FARC y la estructura normativa y conceptual del reformismo agrario vigente en Colombia desde comienzos del siglo XX. Y, finalmente, formula algunas conclusiones y recomendaciones sugiriendo que el preacuerdo agrario confirmaría que las partes en conflicto habrían luchado por decenas de años estando de acuerdo en que deben seguir practicando todo lo que ha fracasado en política agraria conduciendo a la guerra para, por esa misma ruta, terminarla.
\end{abstract}

Palabras clave: concordancias, minifundios, factibilidad, preacuerdos relevantes, reforma agraria.

1. Artículo descriptivo explicativo con enfoques cuantitativo y cualitativo. Resultado de un trabajo desarrollado por sus autores en ejercicio de asesoría a una tesis magistral. Desarrollado en el grupo de investigación Servipúblicos, Universidad Distrital Francisco José de Caldas (Carrera 5 Este

15-82, Bogotá, PBX 2841658) y financiado con recursos de sus autores. Fecha de inicio: agosto 2015. Fecha de terminación, febrero 2016.

2. Economía, Universidad Nacional de Colombia. Maestría Ciencia Política, Universidad de los Andes. Profesor tiempo completo, Universidad Antonio Nariño, Bogotá, Colombia. (Calle 58 A 37-94, Bogotá, PBX 2213257). Docente- investigador del grupo Servipúblicos, Universidad Distrital Francisco José de Caldas de Bogotá, Colombia, Carrera 5 Este 15-82, Bogotá, PBX 2841658, (Reconocimiento Colciencias 2015-2017). Bernardo. congote@uan.edu.co

3. Ingeniero catastral y geodesta doctorante de Ingeniería de la Universidad Distrital. Magister en Planeación Urbana y Regional. Universidad Javeriana. Docente director del grupo de investigación Servipúblicos. Universidad Distrital Francisco José de Caldas de Bogotá, Colombia, Carrera 5 Este 15-82, Bogotá, PBX 2841658. jalizcanoc@udistrital.edu.co 


\begin{abstract}
The colombian civil war should not have any structural justification at least in agrarian problems solutions. The article demonstrates it analyzing the Number One point of the preliminary agreement between the Colombian government and the insurgent faction FARC, named "Política de desarrollo agrario integral". The basic hypothesis exposes the risk of this agreement in maintain the actual smallholding agrarian exploitations and so the structural contradictions to maintain rural poverty which conduced to political violence. First it compares the text of the pre agreement with legal agrarian reforms Laws 200 of 1936 and 135 of 1961 defining their levels of concordance. Second, it demonstrates the lack of any cualitative or cuantitative differences between this preliminary agreement and the principal legal and conceptual agrarian reforms which has been applied (and failed) in Colombia from the begining of the 20 century. So, it generates some conclusions and recomendations suggesting that this paradox should demonstrate that colombian civil war between Stablishment and Insurgence did not have any structural opposition about this essential agrarian policies because both of them agrees in repeating all the historical failed agrarian strategies actions which conduced to the civil war as well as this is presented as the way to negotiate the war's ending.
\end{abstract}

Keywords: Agrarian reform, Concordances, Feasibility, Relevant pre agreements, Smallholdings.

El artículo ofrece análisis cualitativos sugiriendo que sostener el tamaño minifundista de las unidades de explotación agropecuaria ha incidido negativamente en el desarrollo agrario, por tanto, también en el urbano $y$, por esta ruta, en la convivencia pacífica. De esta forma, alerta en torno a algunos riesgos económicos y políticos del denominado preacuerdo agrario Gobierno-FARC en La Habana, que pondrían en riesgo la factibilidad del acuerdo de paz.

El artículo se propone demostrar que el acuerdo preliminar de paz suscrito entre Gobierno y FARC en La Habana (Gobierno de Colombia y Fuerzas Armadas Revolucionarias de Colombia (FARC, 2014), correría el riesgo de contribuir a agravar, no a resolver, el ya precario y violento bienestar rural, debido a que mantiene como solución la misma causa del problema:sustituiro distribuir latifundios para seguir reduciéndolos a explotaciones agrarias minifundistas. Todo ello disfrazado bajo el eufemismo del incentivo a la economía campesina, familiar y comunitaria confundiendo, hoy como ayer, tenencia con productividad de la tierra y economía familiar (léase: minifundista) con bienestar (Berry, 2002, 2013, 2015; Gobierno et al. 2014).

En este orden de ideas, las partes del conflicto estaríamos al borde de asumir compromisos que solo vendrán a reproducir los sofismas que han conducido al fracaso del reformismo agrario en Colombia, de modo que, buscando una paz sostenible, estaríamos, al contrario, asegurando las vigas que contribuyeron a generar el conflicto desde el temprano siglo XX (Currie, 1968 ; Sánchez \& Meertens, 2000).

\section{Análisis del preacuerdo agrario Gobierno - FARC}

\section{Contenidos específicos}

Las guerrillas son 'conservadoras'... por largo tiempo, la opción más fácil es (sic) más de lo mismo... Las FARC son más un movimiento rural que un movimiento agrario... nunca han tenido un programa detallado...

(Y) la visión metropolitana del campo

(en cabeza del régimen) es frecuentemente contradictoria...

(Deas, 2015).

Los cuadros siguientes muestran los resultados del análisis del documento calificado como punto uno del preacuerdo de La Habana entre Gobierno y FARC (Gobierno et al., 2014). Este ejercicio precisa el nivel de concordancias existente entre el preacuerdo y las disposiciones agrarias registradas en las leyes 200/36 y $135 / 61$.

Los cuadros siguientes muestran los resultados del análisis del documento calificado como punto uno del preacuerdo de La Habana entre Gobierno y FARC (Gobierno et al., 2014). Este ejercicio precisa el nivel de concordancias existente entre el preacuerdo y las disposiciones agrarias registradas en las leyes 200/36 y $135 / 61^{4}$.
4. Se seleccionaron estas dos leyes primero, para darle al análisis una conveniente perspectiva histórica $y$, segundo porque, a no ser que se pruebe lo contrario, pueden ser dos piezas fundamentales para entender el ayer y el hoy del enfoque reformista del agro en Colombia que se pretende conservar en las negociaciones de paz. 
Tabla 1

Temas relevantes delp reacuerdo preliminar (según frecuencia en el texto)

\begin{tabular}{|c|c|c|c|c|c|}
\hline Prioridad & Tema & $\begin{array}{l}\text { Participación } \\
\text { en el total (\%) }\end{array}$ & $\begin{array}{l}\text { Porcentaje } \\
\text { acumulado }\end{array}$ & Frecuencia & $\begin{array}{l}\text { Clasificación } \\
\text { cientifica del } \\
\text { tema }\end{array}$ \\
\hline 1 & Acceso, distribución, legalización de & $21 \%$ & $21 \%$ & $12 \mathrm{E}$ & conómica \\
\hline 2 & $\begin{array}{c}\text { Promover, garantizar, estabilizar ingresos } \\
\text { rurales }\end{array}$ & $13 \%$ & $34 \%$ & 7 & Sociológica \\
\hline 3 & $\begin{array}{l}\text { Estimular, reactivar, sostener la } \\
\text { economia campesina, familiar y } \\
\text { comunitaria }\end{array}$ & $11 \%$ & $45 \%$ & 6 & $\begin{array}{l}\text { Politicas } \\
\text { Públicas }\end{array}$ \\
\hline 4 & $\begin{array}{l}\text { Fomento a la producción/productividad de } \\
\text { alimentos }\end{array}$ & $9 \% 5$ & $4 \% 5$ & D & erecho \\
\hline 5 & $\begin{array}{l}\text { Garantizar bienestar rural / Provisión de } \\
\text { bienes y servicios sociales }\end{array}$ & $7 \% 6$ & $1 \% 4$ & I & ngenierías \\
\hline 6 & $\begin{array}{l}\text { Tecnologias, y Innovacioción, Calialdad en la } \\
\text { economia campesina, familiar y } \\
\text { comunitaria }\end{array}$ & $5 \% 6$ & $6 \% 3$ & c & iencia Política \\
\hline 7 & $\begin{array}{l}\text { Comunitaria } \\
\text { Apoyo asociatividad/ Economa solidaria } 5\end{array}$ & $\%$ & $71 \%$ & 3 & Sociológica \\
\hline 8 & Erradicar/reducir pobreza & $4 \% 7$ & $5 \% 2$ & & $\begin{array}{l}\text { Politicas } \\
\text { Púlicas }\end{array}$ \\
\hline $\begin{array}{l}9 \\
10 R \\
11 C\end{array}$ & $\begin{array}{l}\text { Disminuir brechas campo-ciudad } \\
\text { eforma Rural Integral (RRI) } 4 \\
\text { omercialización de productos } 4\end{array}$ & $\begin{array}{l}4 \% 7 \\
\% \\
\%\end{array}$ & $\begin{array}{l}9 \% 2 \\
82 \% \\
86 \%\end{array}$ & $2_{2}^{E}$ & $\begin{array}{l}\text { Publicas } \\
\text { conómica } \\
\text { Derecho } \\
\text { Económica }\end{array}$ \\
\hline $12 \mathrm{C}$ & obro y recaudo de impuestos 4 & $\%$ & $89 \%$ & 2 & $\begin{array}{l}\text { Politicas } \\
\text { Públicas }\end{array}$ \\
\hline $13 \mathrm{~s}$ & istema alimentación / nutrición & $4 \% 9$ & $3 \% 2$ & & $\begin{array}{l}\text { Políticas } \\
\text { Públicas }\end{array}$ \\
\hline $\begin{array}{l}14 \mathrm{C} \\
15 \mathrm{P} \\
16 \mathrm{Z} \\
17 \mathrm{O}\end{array}$ & $\begin{array}{l}\text { onstrucción de paz } 2 \\
\text { reservación del medio ambiente } \\
\text { onas de Reserva Campesina } 2 \\
\text { ferta riego y drenaje rural } \\
\text { TOTAL, DE ITEMS RELEVANTES } 5\end{array}$ & $\begin{array}{r}\% \\
2 \% 9 \\
\% 9 \\
2 \% 1\end{array}$ & $\begin{array}{l}95 \% \\
6 \% 1 \\
98 \% \\
00 \%\end{array}$ & $\begin{array}{l}1 \\
1 \\
1 \\
1 \\
6\end{array}$ & $\begin{array}{l}\text { Ciencia Política } \\
\text { ngenierias } \\
\text { Derecho } \\
\text { Ingenierias }\end{array}$ \\
\hline
\end{tabular}

De la Tabla 1 se puede inferir, entre otros asuntos, que:

- Existen diecisiete (17) temas relevantes en el preacuerdo.

- El de mayor relevancia es el de acceso, distribución, legalización de tierras (21\%).

- El $54 \%$ de los temas preacordados se concentra entre los siguientes cuatro (4) (acceso, distribución, legalización de tierras (21\%); le siguen: promover, garantizar, estabilizar ingresos rurales (13\%); estimular, reactivar, sostener la economía campesina, familiar y comunitaria (11\%) y fomentar la producción / productividad de alimentos (9\%).

- Los cuatro temas más frecuentes coinciden con la temática central que ha estado presente en la discusión y tratamiento de la problemática agraria no sólo en Colombia sino en América Latina desde comienzos del siglo pasado.

- Los siguientes seis (6) son temas considerados generalmenteneurálgicosenladiscusiónsocioeconómica y política actual, pero que, paradójicamente, no registran importancia relevante en el preacuerdo. Son ellos: erradicar o reducir la pobreza; disminuir brechas campociudad; comercialización de productos; construcción de paz; preservación del medio ambiente: oferta de riego y drenaje rural ( $3 \%$ cada tema).

- Llama la atención en particular, que la construcción de paz hubiera obtenido un solo registro en todo el documento (si bien se puede dar por entendida en los demás). Igualmente, que un asunto de la mayor importancia en la discusión moderna del problema agrario como es la disminución de la brecha entre campo y ciudad y la comercialización racional de alimentos, se halle entre los menos relevantes. Fenómeno que confirma parcialmente que el problema agrario, estructuralmente socioeconómico, se sigue manejando utilizando herramientas subjetivas alejadas de sus patrones teóricos básicos.

- Temas que adquirieron relevancia intermedia: garantizar bienestar rural mediante la provisión de bienes y servicios sociales (7\%); ofrecer tecnología, innovación, calidad a la economía campesina, familiar y comunitaria; apoyar la asociatividad o economía solidaria (5\% cada uno).

\section{Conexiones entre el preacuerdo y la tradición reformista agraria en Colombia}

\section{Conexiones con la legislación agraria observada}

[El preacuerdo agrario] exigirá profundos cambios de mentalidad en la sociedad colombiana sobre lo que debe ser el futuro agrario del país, pero... leves modificaciones al derecho agrario vigente...

(Restrepo, 2015).

Tabla 2

Conexiones entre el preacuerdo y las leyes 200/36 y 135/61

\begin{tabular}{|c|c|c|}
\hline Tema relevante del preacuerdo $D$ & $\begin{array}{l}\text { isposiciones conexas } \\
\text { con la Ley } 200 \text { de } \\
1936 \\
\text { Articulo(s) donde } \\
\text { reposan referencias } \\
\text { taxativas al tema }\end{array}$ & $\begin{array}{l}\text { Disposiciones conexas con la Ley } 135 \text { de } \\
\qquad 1961 \\
\text { Articulo(s) donde reposan referencias } \\
\text { taxativas al tema (i) }\end{array}$ \\
\hline $\begin{array}{l}\text { Acceso, distribución, I egalización de } \\
\text { tierras }\end{array}$ & $\begin{array}{l}3,8,12,16,17,18,19 \\
21,22,23,24\end{array}$ & $\begin{array}{l}\text { 1(1), 1(4), 3(a), } 3(\mathrm{c}), 3(\mathrm{f}), 13(\mathrm{~b}), 13(\mathrm{c}), 22, \\
25,29,35, \text { Capitulo I X(xi), Capitulo X(xiii), } \\
\text { Capitulo XI(xiv), C apitulo XVI(xvi),105(b) } \\
\text { (xix). }\end{array}$ \\
\hline $\begin{array}{l}\text { Promover, garantizar, estabilizar } \\
\text { ingresos rurales }\end{array}$ & 141 & \\
\hline $\begin{array}{l}\text { Estimular, } r \text { eactivar, } \\
\text { economia sostener I } \\
\text { comunitaria }\end{array}$ & $1,2,3,14$ & $\begin{array}{l}\text { 1(2), 1(4),29, 32, 35, C apitulo IX (xi),46, } \\
\text { Capitulo X (xiii), Capitulo XI(xiv),80, Capitulo } \\
\text { XVI(xvi), Capitulo XVII(xvii),195(xix), }\end{array}$ \\
\hline $\begin{array}{l}\text { Fomento a la producción/productividad } \\
\text { de alimentos (xii) }\end{array}$ & Ninguno & 1(2), 1(3), $3(\mathrm{e}), 3(\mathrm{~h}), 105(\mathrm{xix})$ \\
\hline $\begin{array}{l}\text { Garantizar bienestar } r \text { ural / P rovisión } \\
\text { de bienes y servicios sociales }\end{array}$ & Ninguno & $\begin{array}{l}\text { 1(5), 3(e), 3(j),19, Capitulo XII, Capítulo XVII } \\
\text { (xvii), }\end{array}$ \\
\hline $\begin{array}{l}\text { Tecnologia, innovación, crédito, calidad } \\
\text { en I a economia campesina, familiar y } \\
\text { comunitaria }\end{array}$ & 141 & $\begin{array}{l}\text { (5), } 3 \text { (h). } 3 \text { (j), Capitulo V(viii), Capítulo XIII } \\
\text { (xv), Capitulo XVII(xvii), }\end{array}$ \\
\hline $\begin{array}{l}\text { Apoyo asociatividad/ Economía } \\
\text { solidaria }\end{array}$ & Ninguno & $\begin{array}{l}\text { 1(5), 3(k), Capitulo I X (xi),80, C apitulo } \\
\text { XVII(xvii), Capitulo XVIIII, }\end{array}$ \\
\hline Erradicar/reducir pobreza $\mathrm{N}$ & inguno & 1(5) \\
\hline Disminuir brechas campo-ciudad $\mathrm{N}$ & inguno & Ninguno \\
\hline Reforma Rural Integral (RRI) & Ninguno & 9 \\
\hline Comercialización de productos & Ninguno & 1(5), 3 (e), 3(h), Capítulo XVII(xvii) \\
\hline Cobro y recaudo de impuestos & Ninguno & 3(d)(ii), Capitulo V (viii) \\
\hline Sistema alimentación / nutrición & Ninguno & 9 (v), Capitulo XVIII, \\
\hline Construcción de paz $\mathrm{N}$ & inguno & 13(a) (vi), 13 (d)(vii), $105(x v i i i), 110(x x)$ \\
\hline Preservación del medio ambiente (ix) 5 & , 9,10,13 & $1(6), 3(\mathrm{c}), 3(\mathrm{f}), 3(\mathrm{~g}), 19,39$ \\
\hline Zonas de Reserva Campesina N & inguno & $\begin{array}{l}3 \text { (i) (iii), } 3 \text { (k) ( iv),40 (x), Capitulo IX (xi), } \\
\text { Capitulo X (xiii),80, C apitulo XVI(xvi), } 105 \text { (b) } \\
\text { (xx) }\end{array}$ \\
\hline Oferta riego y drenaje rural & 9 & 1(5), 3(f), 3(h),19, Capitulo XII, \\
\hline
\end{tabular}

Fuente

Gobierno et. al., (2014). Ley 200 de 1936. Ley 135 de 1961. Análisis del artículo5. Ver también: Berry (2002)

5. El artículo se ampara en el beneficio del error humano para explicar posibles errores $\mathrm{u}$ omisiones en lo relativo al exhaustivo trabajo comparado de este análisis. 


\section{Pies de cuadro}

Para facilitar el análisis se incluyen aquí los veinte pies de la Tabla 2.

\section{Tabla 3}

\section{Pies de página de la Tabla 2}

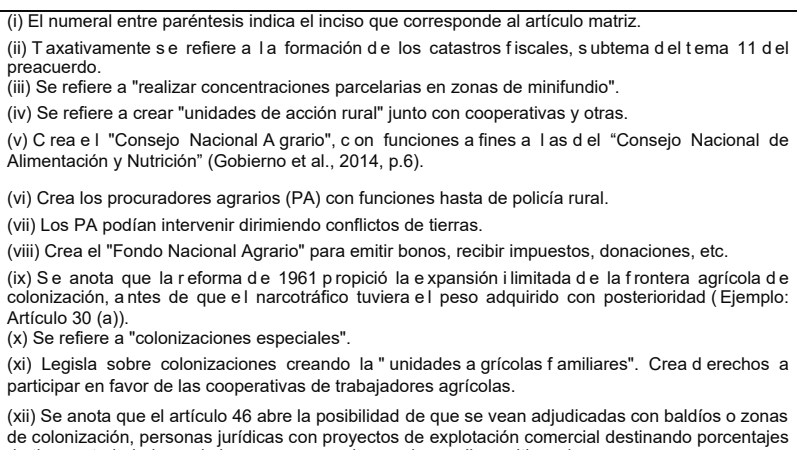

(xiii) Legisla detalladamente sobre la "unidades agrícolas familiares".

(xiv) $\mathrm{E}$ I preacuerdo $\mathrm{se}$ refiere a que el Fondo de $\mathrm{T}$ ierras $\mathrm{se}$ e alimentará de tierras I egalmente adquiridas, cuyo $p$ erfil e stá ya también reglamentado e $n$ este $c$ apítulo de l a reforma d el 61 (Gobierno et al., 2014, p.7).

(xv) Financiación de las entidades ejecutoras de la reforma agraria.

(xvi) Reglamenta todo lo atinente al minifundio como propiedad igual a inferior a 3 hectáreas que se declara indivisible. Ver especialmente el artículo 90 hablando de explotaciones de "superficie

(xvii) Reglamenta la provisión de bienes y servicios sociales en el campo.

(xviii) Destina recursos de Fuerzas Armadas para entrenar campesinos en el manejo de maquinaria agrícola, y otras labores de producción agropecuaria.

(xix) Dispone reasignación de unidades agrícolas familiares a miembros de Fuerzas Armadas.

(xx) Conexo con papel de los PA (pies de página vi y vii).

\section{Fuente}

Tabla 2 y artículo

\section{Conclusiones preliminares}

\section{Del análisis comparado se puede concluir:}

\section{En lo específico}

La Figura 1 presenta una radiografía de los niveles de conexidad entre el preacuerdo actual y lo dispuesto en las leyes agrarias 200/1936 y 135/ 1961.

Se observan aquí, entre otros hechos, los siguientes:

- La estrecha conexidad entre el preacuerdo de acceso a la tierra y el número de veces en que el ítem, también mayoritariamente, se inscribió en las leyes 200/36 once (11) y 135/61 diecisiete (17) veces. En paralelo, dicha conexidad se cumple también con el fomento a la economía campesina, sinónimo de fomento al minifundio inscrita en la ley 200/36 cuatro (4) y en la 135/61 trece (13) veces.

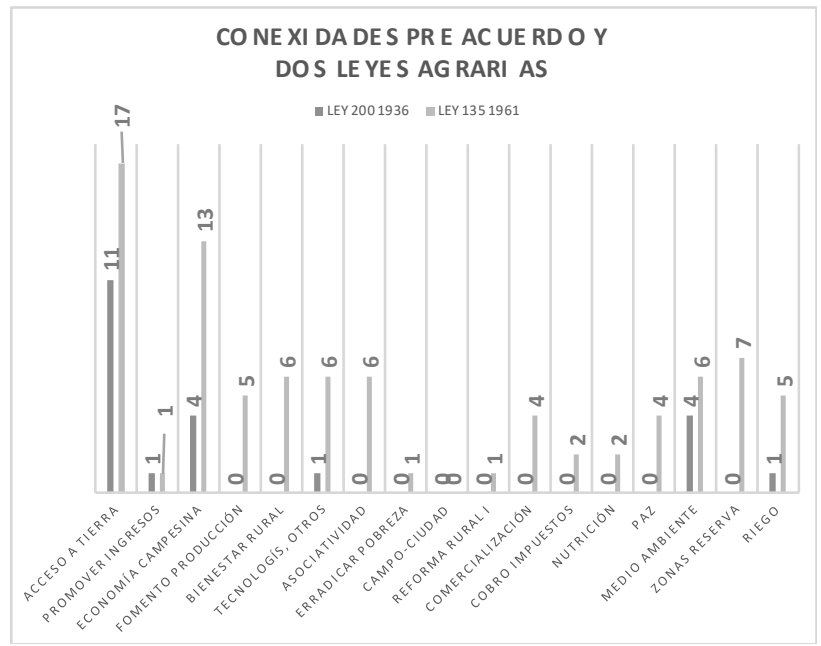

Figura 1.

Conexidades entre el preacuerdo Gobierno- FARC y las reformas agrarias (Método: nube de puntos)

- Los otros ítem de mayor conexidad con ambas leyes son, en su orden (y frecuencia):

- Protección del medio ambiente (10 veces).

- Suministro de tecnología y otros (7 veces).

-Zonas de Reserva Campesina (7 veces).

- Bienestar rural y Estímulo a la asociatividad (6 veces c/u).

- Los ítems de mayor conexidad con lo dispuesto en la ley 200 , son en orden (y frecuencia):

- Acceso a la tierra (11)

- Fomento a la economía campesina y Protección del medio ambiente $(4 \mathrm{c} / \mathrm{u})$.

- Los ítems de mayor conexidad con lo dispuesto en la ley 135 , son en orden (y frecuencia):

- Acceso a la tierra (17)

- Fomento a economía campesina (minifundio) (13)

- Zonas de Reserva Campesina (7).

- Bienestar rural, Suministro de tecnología y otros, Estímulo a la asociatividad y Protección del medio ambiente $(6 \mathrm{c} / \mathrm{u}) \mathrm{y}$

- Fomento a la producción y Oferta de riego (5). 
- Comercialización de productos y Construcción de paz (4).

\section{En lo general}

- Dieciséis (16) de los diecisiete (17) temas relevantes del preacuerdo Gobierno-FARC (94\%), tienen relación de conexidad con por lo menos una disposición de las reformas agrarias de 1936 o de 1961.

- El único tema relevante del preacuerdo que no halla correspondencia con la legislación precedente, es el relacionado con disminuir la brecha campo-ciudad. Esta desconexión confirma que, siendo una forma clave para explicar y tratar el problema agrario, su bajo peso ratifica que elementos esenciales del debate temático ni ayer ni hoy han formado parte de las visiones del reformismo agrario en Colombia.

- La conexidad más importante observada entre los tres documentos analizados radica en que el acceso a la tierra es, al tiempo, Prioridad 1 de los preacuerdos y de las leyes $200 / 36$ y 135/1961. En paralelo, el fomento al minifundio es, al tiempo, Prioridad 3 de los preacuerdos y Prioridad 2 de las Leyes $200 / 36$ y 135/61.

\section{En lo global}

- La evidente alta conexidad entre los preacuerdos y la legislación analizada en torno al objetivo de facilitar el acceso a la tierra en el agro, configura uno de los argumentos centrales para poner en duda, al menos de manera preliminar, la viabilidad operativa de lo tratado en La Habana en el evento de que pase a ser acuerdo final y, además, logre ser implementado. Por un lado, porque si bien otros estudios han probado las fragilidades teórico-prácticas de ese modelo (Castro, 1972; Congote \& García, 2014; Contreras, 1971; Sandilands, 1971), por el otro, porque podría anticiparse que, hoy como ayer, el Estado se estaría comprometiendo a dedicar ingentes volúmenes de los impuestos de los contribuyentes para buscar soluciones que el tiempo ha probado fallidas para resolver el problema agrario colombiano (Congote et al., 2014).

- Se observa que temas como comercialización, preservación del medio ambiente, construcción de paz, zonas de reserva campesina y oferta de riego, que en las leyes 200/36 y 135/61 fueron tratados con relevancia relativamente alta, en el preacuerdo y figuran en el grupo de los siete temas a los que Gobierno-Farc les dieron la menor relevancia. Ello sugeriría que con el paso de los años el enfoque tiende hacia una arriesgada simplificación, tal vez explicable porque el gran peso de negociar la paz puede inducir a cesiones que, como estas, en estricto sentido o en otro escenario, no habrían sido admisibles. Precisamente por este tipo de señales, también queda en duda la factibilidad de estos preacuerdos dado que, de implementarse, sus ejecutores se toparían con tareas marcadamente subjetivo-políticas antes que económico-prácticas, controversia que sería caldo de cultivo para mantener activas las causas del conflicto armado.

- Aunque la materia que trata el artículo es estrictamente económica, la responsabilidad política de la teoría económica puesta de relieve entre otros por Marx (1857) y Keynes (1936), permite proponer que esta notoria conexidad entre establecimiento e insurgencia que se prueba vigente durante un período que data al menos desde 1936, plantearía otra hipótesis relacionada con la posible inocuidad de una guerra civil interna, cuyas dos denominadas partes habrían luchado por asuntos sobre los cuales siempre habrían estado de acuerdo, a saber: la preservación de la pobreza y violencia rurales aplicando la vieja táctica rentista de buscar "ganancias en río revuelto".

- Los análisis realizados probarían que siendo las económicas, algunas de estas llamadas causas objetivas de la guerra, al demostrarse inoperantes obligarían a voltear la atención acerca, entonces, de qué tipo de causas subjetivas culturales alimentan una beligerancia política que en Colombia trasciende al movimiento insurgente de los años sesenta del siglo XX y nos manda hacia la violencia política de los siglos XIX y XX (Congote, 2004).

\section{Breve evaluación de la factibilidad teórica y práctica del preacuerdo agrario Gobierno - FARC}

\section{Presentación}

Una vez establecida la múltiple conexidad entre Gobierno e insurgencia con la tradición agrarista colombiana, se analiza su pre factibilidad teórica y práctica. Precisando que las partes han ratificado por escrito que estas negociaciones están regidas por el principio de que nada está acordado hasta que todo esté acordado (Gobierno et al., 2014).

Se está probando la no factibilidad de este preacuerdo porque procura resguardar e incentivar la explotación minifundista del agro (prioridades 1 a 5 del Cuadro 2). Igualmente, utilizando datos de un estudio de caso (investigación magistral) explica los daños colaterales que también puede traer consigo el preacuerdo con base en otras prioridades (P), (P6, P11, P15 y P17 de Tabla 
2; Congote et al., 2014; García, 2011).

\section{Hipótesis a evaluar}

El artículo evalúa la factibilidad del ítem de mayor conexidad y relevancia del preacuerdo, según el cual las partes decidirían que la que llaman "Política de Desarrollo Integral" o "Reforma Rural Integral", consista en seguir facilitándoles a los habitantes del campo el acceso a la tierra en tamaños y modos de explotación minifundistas $y$, al tiempo, pretender que puede garantizar tanto la elevación de la producción como la estabilidad de los ingresos rurales y el bienestar zonal, entre otros, mediante la implementación de tecnología y sistemas de almacenamiento y comercialización de productos.

\section{Algunos posibles resultados}

Sobre la factibilidad preliminar de los cinco ejes prioritarios del preacuerdo

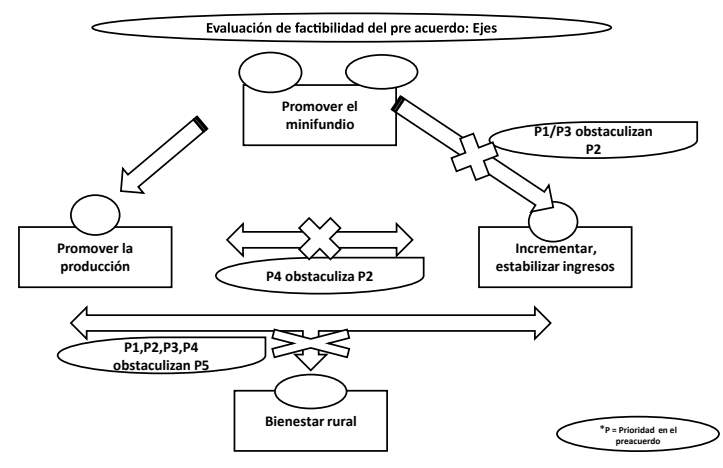

Figura 2.

Evaluación de factibilidad de los ejes del preacuerdo. Adaptado de los cuadros 2 y 3 del presente artículo

$\mathrm{Si}$ se clasifican como ejes prioritarios los cinco preacuerdos que acumularon en el análisis el $51 \%$ de las frecuencias (prioridades 1 a 5), se puede proponer este análisis de factibilidad:

- La promoción de la tenencia de tierra bajo modalidades familiares, equivale a la promoción y protección del modo de explotación minifundista (P1 y P3).

- La modalidad minifundista de explotación, si bien puede aumentar la producción, no garantiza ni el incremento ni la estabilidad de los ingresos de los habitantes del agro (P2) ni tampoco la productividad medida como producción / combinación del empleo de todos los factores o recursos involucrados. (Congote et al., 2014; Contreras, 1971; Sandilands, 1971).
- El estímulo a la producción agropecuaria bajo el modo de explotación minifundista (P4) también atenta contra la posibilidad de incrementar o estabilizar los ingresos (P2), debido a que esta correlación está bloqueada por el principio de las elasticidades precio e ingreso de la demanda por bienes agropecuarios según el cual cualquiera sea el volumen de producción agropecuario, su demanda efectiva está limitada sea por la capacidad biológica del consumo humano, por las limitaciones existentes para conservar alimentos durante largos plazos o por las complejidades implícitas en exportar excedentes (Congote et. al., 2014; Currie, 1968; García, 2011).

- La suma de colisiones entre las prioridades 1 a 4, bloquearía la factibilidad de que, por esta ruta, se pueda garantizar el bienestar rural soñado por el preacuerdo (P5) y, por esta ruta, que se perpetúen las causas tradicionales de la violencia política.

Sobre los daños colaterales que genera la no factibilidad de los ejes prioritarios del preacuerdo

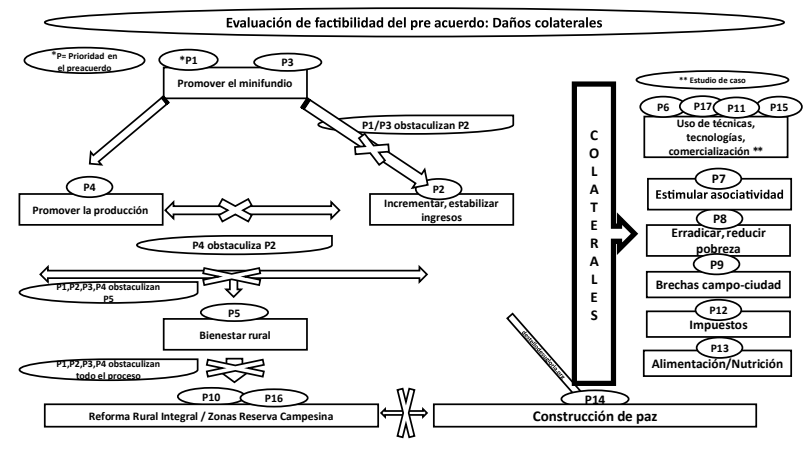

Figura 3.

Evaluación de los daños colaterales del preacuerdo

La promoción de un modo de explotación minifundista, amén de los obstáculos que establece contra las prioridades 3,4 y 5 del preacuerdo, induciría daños colaterales que pondrían en duda también la factibilidad de que se puedan implementar exitosamente los demás ítems del preacuerdo, dado que:

- Impide que se puedan destinar eficientemente recursos para aplicar técnicas y tecnologías innovadoras en el campo (P6 y P11) y, de paso, que se puedan ofrecer facilidades de almacenamiento, comercialización o financiación exitosas a los tenedores de este tipo de explotaciones $y$, menos, garantizar la adecuada protección al medio ambiente, tal como lo proponen las prioridades 15 y 17 (Congote, 2011a; Congote et al.; 2014; García, 2011; Contreras, 1971). 
- El hecho de que, particularmente la reforma agraria de 1961, haya abundado en disponer un grueso y costoso mecanismo institucional para apalancar objetivos múltiples como los que plantea el preacuerdo (creación del Incora, el Consejo Nacional Agrario, los procuradores agrarios y el Fondo Nacional Agrario, entre otros), sin que cincuenta y cuatro años después el agro colombiano registre cambio sustancial alguno frente a su situación previa (llámese cambios en la pobreza relativa en el campo, subempleo de mano de obra y tierras, violencia rural, inequitativa distribución de tierras, etc.) refuerza la probatoria válida de la hipótesis (Ver Cuadro 3. Ver también: Faguet et al., 2015) .

- Igualmente, obstaculiza la posibilidad de que se pueda estimular la asociatividad cooperativa, disminuir la pobreza, cerrar las brechas campo - ciudad, recaudar o pagar impuestos o generar programas exitosos de mejoría alimentaria (P7, P8, P9, P12 y P13) (García, 2011).

- Esta delicada combinación de ejes obstaculizadores y daños colaterales, no sólo pone en duda a fondo la viabilidad de que el preacuerdo alcance sus metas de política orientadas a que mediante la que mal llama "reforma agraria integral", se logre construir la paz en las zonas agrícolas minifundistas de Colombia (P10, P16 y P14) sino que, tampoco lo lograría en las zonas no minifundistas porque el preacuerdo ha omitido formular planteamientos coherentes sobre el estado de este modo de explotación en Colombia .

- Este análisis podría servir de base para poner en duda, preliminarmente también, la factibilidad política del preacuerdo, en la medida en que, primero, demostrada la conexidad temática del preacuerdo entre Gobierno e insurgencia, ella estaría evidenciando nada menos que la inocuidad de la guerra civil dado que las partes siempre habrían estado de acuerdo en aplicar soluciones fracasadas al problema del campo. $Y$, segundo que, definidos los ejes prioritarios del preacuerdo como altamente conexos con parte de la legislación agraria más importante del siglo XX, las leyes 200/1936 y 135/1961, el preacuerdo acusaría evidente fragilidad al proponerse cumplir metas que durante casi un siglo han mostrado ser ilusorias y, peor generadoras de frustración colectiva en el campesinado induciéndolo a incurrir en el delito como forma de supervivencia.

\section{Conclusiones}

Locura: hacer siempre lo mismo y esperar resultados diferentes.
Primera. Las observaciones del artículo validan preliminarmente la hipótesis de que el preacuerdo Gobierno - FARC de La Habana, concentrado en fortalecer el minifundio bajo el eufemismo del impulso a la denominada economía campesina, familiar y comunitaria, no resulte factible de llegar a convertirse en acuerdo definitivo y, por tanto, en política pública.

Segunda. Durante buena parte de los últimos ochenta años Colombia ha implementado diversas reformas agrarias que, como la 200/36 y la 135/61, repiten fórmulas cuyos resultados se han mostrado fallidos, tanto como que ellos mismos han sido aducidos como causantes del clima de violencia imperante en el agro (Currie, 1968; Sánchez, 1985, 1999, 2000). Este mismo vicio, al aparecer evidenciado en el preacuerdo Gobierno - FARC, anuncia que sus resultados también serán negativos.

Tercera. El preacuerdo se concentraría en resolver las contradicciones de las zonas de minifundio aplicando fórmulas probadamente fallidas, algunos de cuyos datos comprueban la reproducción indetenible del minifundio que trae consigo daños que impactan negativamente la prosperidad socioeconómica rural al disminuir los ingresos (nominales y reales), elevar el submpleo familiar $y$, por esta ruta, conservar la calidad rondando el nivel mínimo de necesidades básicas satisfechas (Congote et al., 2014).

Cuarta. El preacuerdo diseña una estrategia prominifundio cuyos daños colaterales son múltiples, impidiendo, por ejemplo, que sea viable aplicar en esos territorios herramientas técnicas, tecnológicas, de mercadeo y asociativas que, vistas en términos absolutos son, de suyo, defendibles. Prueba fáctica de lo anterior es que habiendo sido establecidos todos estos patrones en las leyes 200/1936 y 135/1961, decenas de años después la insurgencia campesina en La Habana sigue reclamándolos por su ausencia, pero al tiempo, termina unida a la política que garantiza seguir aplicándolas, en un juego que raya con el desquiciamiento conceptual.

Quinta. El preacuerdo agrario debería renombrarse. Llamado ahora "Política de desarrollo integral" y hasta "Reforma agraria integral", se reduce a una suma de fórmulas contradictorias cuya factibilidad teórica y práctica ha sido puesta en duda por los hechos históricos. Al final, sólo se reduce a resolver y mal, problemas del minifundio que caracteriza a unas pocas regiones andinas de Colombia, dejando sin examen, por ejemplo, el tratamiento de las vastas regiones de la Altillanura y los Valles del Cauca, Sinú, San Jorge y Tolima - Huila. 
Sexta. Por esta misma ruta, el estímulo al subempleo minifundista de fuerza de trabajo explícito en el modo de producción minifundista, solo profundizaría la expulsión de mano de obra desde el campo hacia la ciudad, arriesgando abrir más la brecha entre estos dos ejes del desarrollo económico y sumando más mano de obra expulsada hacia nuevas formas de violencia, teniendo como final trágico la reproducción de orígenes que, probadamente, también han sido trágicos.

Séptima. Se destaca, negativamente el hecho aquí probado de que, habiendo estado la insurgencia y el establecimiento durante décadas de acuerdo en el qué y cómo hacerlo (ambos víctimas de graves errores), se haya derramado tanta sangre y minado la esperanza nacional por décadas para lograr tan poco, lo que obligaría a buscar la explicación de esta guerra mucho más allá de estas que se suelen llamar, benignamente, causas objetivas.

\section{Recomendaciones}

Primera. El artículo, de manera preliminar la conveniencia de someter a crítica la factibilidad teórica y práctica del punto uno del acuerdo político FARC - Gobierno o, en el peor de los casos, la conveniencia de advertir acerca de sus múltiples riesgos.

Segunda. El panorama del preacuerdo agrario está restringido solo a algunas regiones y modos de explotación agraria en Colombia, razón por la cual sugerimos que, para evitar frustraciones adicionales, convenga que a escala del acuerdo final se precise el carácter limitado, ya no solo conceptual y político sino geográfico, del que adolece.

Tercera. Omitir alguna de estas acciones podría conducir a que nuevas frustraciones alimenten una vez más la proclividad violenta de las masas campesinas históricamente afectadas por las improvisaciones del establecimiento, ahora en connivencia con la insurgencia. Cuarta. El artículo advierte finalmente que lo observado pueda ser útil para anticipar algunos riesgos implícitos también en la política de restitución de tierras que, aplicada desde 2011, amenaza con aumentar irracionalmente la oferta agrícola, profundizando algunos de los círculos viciosos aquí expuestos.

Quinta. Resultaría saludable que, ante la cercanía de otra negociación Gobierno - ELN, estas conversaciones pudieran subsanar los graves errores denunciados en este documento en el caso de que la advertencia aparezca tarde en lo relacionado con las FARC.

\section{Agradecimientos}

Reconocimientos del autor principal al impulso académico e investigativo recibido del coautor, MSc José A Lizcano; al grupo de investigación Servipúblicos de la Universidad Distrital Francisco José de Caldas y al trabajo investigativo del hoy MSc Jaime García, docente de la Universidad Pedagógica y Tecnológica de Colombia, cuya asesoría de tesis por parte del autor principal, trajo consigo algunas de las semillas de este documento.

\section{Referencias}

Berry, A. (2002). ¿Colombia encontró por fin una reforma agraria que funcione? Revista de Economía Institucional, 4 (6), pp. 25-70.

Berry, A. (2013). Se necesita un gran cambio a favor de la pequeña agricultura en Colombia. Cali. Recuperado de www.eltiempo.com

Berry, A. (2015). ¿Cómo acabar con la pobreza y bajar la desigualdad? (Ponencia en el foro sobre el Desarrollo Social para el post conflicto). Bogotá: Universidad Jorge Tadeo Lozano. Recuperado de www.utadeo.edu.co

Castro, P. (1972). Un campesino previno al país. Bogotá: Tercer Mundo.

Congote, B. (2004). Anatomía religiosa de la guerra (Tesis de Maestría). Universidad de los Andes. Bogotá.

Congote, B. (2011a). "Tres lecturas sobre la depredación del ecosistema. El caso de Colombia". Revista Tecnogestión, 8, pp. 67-81.

Congote, B. \& García. J. (2014). Dos hipótesis sobre el efecto que tiene en la forma de explotación agropecuaria aplicar subsidios a sistemas de riego. El caso del Alto Chicamocha (Boyacá, Colombia). Bogotá: Revista Cuadernos de Economía 33 (62), enero-junio de 2014.

Congreso de la Republica de Colombia. (diciembre 16 de 1936) Sobre régimen de tierras. [Ley 200 de 1936].

Congreso de la Republica de Colombia. (diciembre 20 de 1961) Sobre reforma social agraria. [Ley 135 de 1961].

Contreras, S. (1971). “Política agraria colombiana. Crítica a la luz del método de Marx y del concepto de racionalidad económica". Bogotá: Universidad Nacional de Colombia. Currie, L. (1968). Desarrollo económico acelerado. México, D. F.: FCE. 
Deas, M. (2015). (21 de julio de 2015). "Sobre la paz". Diario El Tiempo. Recuperado de http://www.eltiempo. $\mathrm{com} /$

Faguet, J., Sánchez, F. y Villaveces, J. (2015). “Land Reform, Latifundia and Social Development at Local Level in Colombia, 1961-2010". Bogotá: CEDE (06).

García, J. (2011). Perspectivas del desarrollo en la región del Alto Chicamocha (Tesis de maestría). Universidad Pedagógica y Tecnológica de Colombia (UPTC),Tunja.

GobiernodeColombiay Fuerzas Armadas Revolucionarias de Colombia (FARC). (2014). Informe Conjunto de la mesa de conversaciones entre el Gobierno de Colombia y las Fuerzas Armadas Revolucionarias de Colombia - Ejército del Pueblo, FARC-EP. Bogotá: periódicos de mayor circulación nacional.

Keynes, J., (1970) [1936]. Teoría general de la renta, el interés y el dinero. México: FCE.
Marx, K. (1989) [1857]. Introducción a la crítica de la economía política. Bogotá, Buenos Aires, Madrid, México: Siglo XXI.

Restrepo, J. (2015).La propiedad rural. Revista Economía Colombiana. Recuperado de www.contraloriagen.gov.co Sánchez, G. (1985). Ensayos de historia social y política del siglo XX. Bogotá: Áncora.

Sánchez, G. (1999). "Guerra y política en la sociedad colombiana". Revista de Análisis político, (11).

Sánchez, G. y Meertens, D. (2000). Bandoleros, gamonales y campesinos. Bogotá: Áncora.

Sandilands, R. (1971). "La modernización del sector agropecuario y la migración rural-urbana en Colombia". Revista de Planeación y Desarrollo, 3 (3), pp. 25-57. 